\title{
The author replies
}

Thank you for allowing me to respond to the letter from Dr. $Z$. Cala to the editor regarding our recent article, Videolaparoscopic Implantation of Long-term Peritoneal Dialysis Catheters [3]. The use of laparoscopy for implantation of peritoneal dialysis (PD) catheters has been championed by Stephen Ash, a nephrologist, since 1981 [2]. As reflected by the number of published reports in the surgical literature during the past decade, surgeons have become increasingly involved in developing an expanded role for videoscopic surgery in the care of the PD patient. As exemplified by Dr. Cala's letter, we continue to seek improvement in implantation techniques and to make the procedure safer for these chronically ill patients.

The trocar device described by Dr. Cala has the potential economic advantage of being a reusable system. Like other appliances specifically designed for PD catheter implantation $[1,2]$, it provides a safe and relatively atraumatic conduit to the peritoneal cavity. Unfortunately, none of the previously described appliances provide adequate airtight seals, thereby making it difficult to maintain a satisfactory pneumoperitoneum during insertion of the PD catheter. Dr. Cala did not address this point in the description of his trocar, however. Evaluation of Figure 1 does not disclose the presence of a valve mechanism. In my opinion, the InnerDyne port (InnerDyne, Salt Lake City, UT, USA) currently remains the best access device for implanting PD catheters. Its spring-loaded pneumoperitoneum needle with overlying expandable sleeve provides exceptional control as it is passed through the rectus sheath. Although the port reducer cap of the $7 / 8$ cannula must be modified by incising it at two points $180^{\circ}$ apart to permit the passage of the Dacron cuffs, it maintains an airtight seal around the catheter during the insertion process.

One important difference between the procedure as described by Dr. Cala and ours is that we are able to perform the laparoscopic implantation with the patient under local anesthesia using minimal or no sedation. This overcomes one of the greatest concerns regarding this method because it avoids subjecting these oftentimes severely ill patients to the risk of a general anesthetic. Initially, we were able to accomplish this with the use of nitrous oxide gas as our insufflation agent because, unlike carbon dioxide $\left(\mathrm{CO}_{2}\right)$ gas, it is painless and devoid of metabolic consequences. We have since switched to the use of helium gas, which is painless, metabolically inert, and noncombustible [4].

Another difference between our procedures is that Dr. Cala uses a three puncture technique, whereas our procedure requires only two puncture sites.
The final location of the deep catheter cuff is unclear from Dr. Cala's procedure description. It should be stressed that the deep cuff should be positioned within the rectus sheath. The rich blood supply of the rectus muscle provides for firm tissue ingrowth and resistance to tunnel infections and pericannular herniation. Just as a superficial cuff may extrude through the exit site, a deep cuff can extrude into the peritoneal cavity. A deep cuff in the relatively loose tissues of the preperitoneal space seems more susceptible to intraperitoneal extrusion and could become the source of relapsing peritonitis.

One advantage of laparoscopic catheter implantation is that it allows the surgeon to address other problems identified during the procedure. As in Dr. Cala's patient series, we have performed adhesiolysis to eliminate lower abdominal compartmentalization, which would have resulted in catheter drainage dysfunction. Also, we have prophylactically tacked up redundant, thin, filmy omentum to the upper abdomen when it was found to be filling the pelvis. On occasion, we have identified and repaired previously unsuspected abdominal wall hernias.

At this writing, we have performed 131 laparoscopic PD catheter implantations. The procedure can be performed safely with the patient under local anesthesia on an ambulatory basis. It significantly reduces the incidence of outflow dysfunction and permits simultaneous identification and correction of previously unsuspected problems that could complicate dialysis therapy. We feel very strongly that laparoscopic PD catheter implantation is the standard of care for clinical practice.

\section{References}

1. Amerling R, Cruz C (1993) A new laparoscopic method for implantation of peritoneal catheters. ASAIO J 39: M787-M789

2. Ash SR, Wolf GC, Bloch R (1981) Placement of the Tenckhoff peritoneal dialysis catheter under peritoneoscopic visualization. Dial Transplant 10: 383-386

3. Crabtree JH, Fishman A (1999) Videolaparoscopic implantation of long-term peritoneal dialysis catheters. Surg Endosc 13: 186-190

4. Crabtree JH, Fishman A (1999) Videoscopic surgery under local and regional anesthesia with helium abdominal insufflation. Surg Endosc 13: 1035-1039

\section{J. H. Crabtree}

Department of Surgery

Southern California Permanente Medical Group

Kaiser Permanente Bellflower Medical Center

9400 East Rosecrans Avenue

Bellflower, CA, 90706, USA 